\title{
Bone transport using the Ilizarov method: a review of complications in 100 consecutive cases
}

\author{
Claudio Iacobellis • Antonio Berizzi • \\ Roberto Aldegheri
}

Received: 30 June 2009/Accepted: 19 February 2010/Published online: 9 March 2010

(C) Springer-Verlag 2010

\begin{abstract}
We present the results of treatment of 100 patients (72 men, 28 women) by the Ilizarov method of bone transport using circular (55) and monolateral external fixators (45). A total of 26 femurs (18 monolateral, 8 circular) and 74 tibias (49 circular, 25 monolateral) was examined. There were no significant differences between the circular fixator and the monolateral fixator with regard to treatment time, complications in the treated bone segments or compliance with the presence of the fixator. The main complications (pseudoarthrosis at bone contact points after transport, insufficient ossification of lengthened bone, knee stiffness) were resolved with further treatment for all patients with the exception of one case which continued with repeated infections. The circular fixator allows for deformity corrections during bone transport but the monolateral fixator is tolerated better by patients, especially in those with femoral defects.
\end{abstract}

Keywords Bone transport · External fixator ·

Distraction osteogenesis

\section{Introduction}

Bone transport according to the Ilizarov method is widely practised in reconstructive surgery. There are many external fixators which are designed to achieve the surgical

C. Iacobellis · A. Berizzi $\cdot$ R. Aldegheri Orthopaedic and Traumatologic Clinic,

University of Padua, Padua, Italy

A. Berizzi $(\bowtie)$

Clinica Ortopedica, Via Giustiniani 2,

35100 Padua, Italy

e-mail: berizzi@tin.it objective of managing bone defects using the principles of distraction osteogenesis. We present our clinical experience of 100 consecutive cases of bone transport (26 femurs, 74 tibias) treated with monolateral and circular external fixators. We include a review of the literature [1-10] and report our results according to the bone segments treated, technical variations, complications and the solutions adopted.

\section{Materials and methods}

Our sample contains 100 patients (72 men, 28 women) with a mean age 34.6 (11-75). There were 55 patients treated with the Ilizarov circular fixator (Amplimedical s.p.a, Milano) and 45 patients treated with the LRS monolateral rail (Orthofix SRL, Verona). A total of 26 femurs (18 treated with the monolateral device and 8 with the circular fixator) and 74 tibias (49 circular fixator, 25 monolateral) were reviewed. A single level bone transport was performed in 80 patients [descending technique in 50 tibias (Figs. 1, 2) and 12 femurs (Fig. 3); ascending technique in 13 tibias and 5 femurs (Fig. 4)], and a double level bone transport in 10 tibias (6 with mid-diaphyseal contact of transported bone ends from proximal and distal metaphyseal osteotomies, and 4 serial "twins" from a double proximal osteotomy) and 5 femurs (all with mid-diaphyseal contact). In 5 cases ( 2 tibias, 3 femurs), we performed a variation of the bone transport technique through immediate shortening by $15 \%$ and subsequent lengthening.

The pathologies causing bone loss, either directly or indirectly from surgical resection, were as follows:

1. Open fractures with loss of bone: 13 tibias, 6 femurs

2. Osteomyelitis: 25 tibias, 4 femurs 


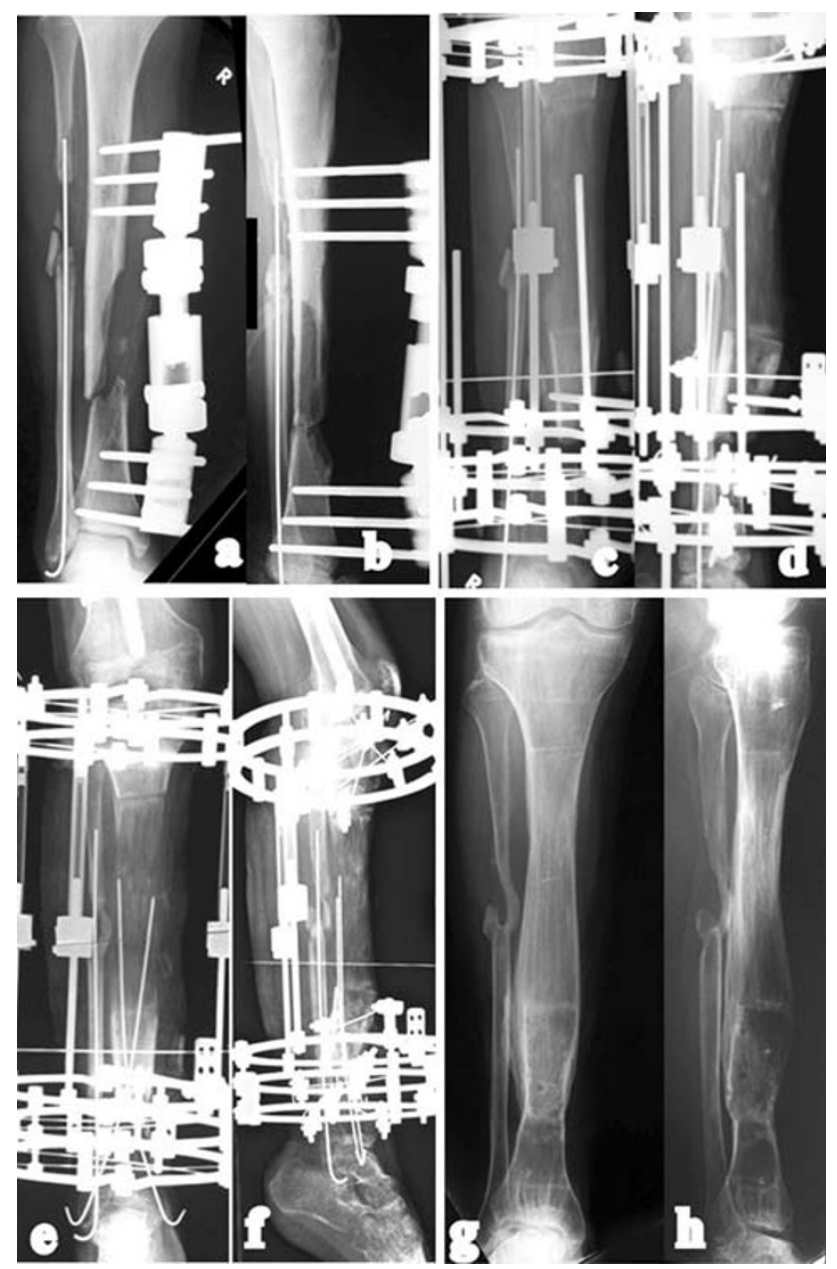

Fig. 1 G.M., man, aged 19. a, b Exposed fracture with bone gap, treated urgently with monolateral fixator. c, d Check-up 5 months after resection and transport. e, f Check-up after second surgical examination of bone ends at point of contact. $\mathbf{g}$, $\mathbf{h}$ Check-up 4 years later

3. Infected nonunions: 32 tibias, 13 femurs

4. Atrophic nonunions: 5 tibias, 2 femurs

The average bone defect after resection was $8.4 \mathrm{~cm}(3-19)$.

Bone transport with the circular external fixator

In the tibia, the assembly comprised of 5 rings: 2 proximal rings, 1 for transport and 2 distal. Two Kirschner wires were inserted and tensioned for each of the proximal and distal rings; 2 screws coated with hydroxyapatite were applied to the ring used for transport (on the medial subcutaneous surface for the tibia and laterally for the femur) in order to reduce skin problems from the crossed wires. In the femur, a mixed assembly with arches and proximal screws was used with 1 ring for transport and 2 distally. The osteotomy was performed with a Gigli saw [11] after the fixator was applied. Bone transport was
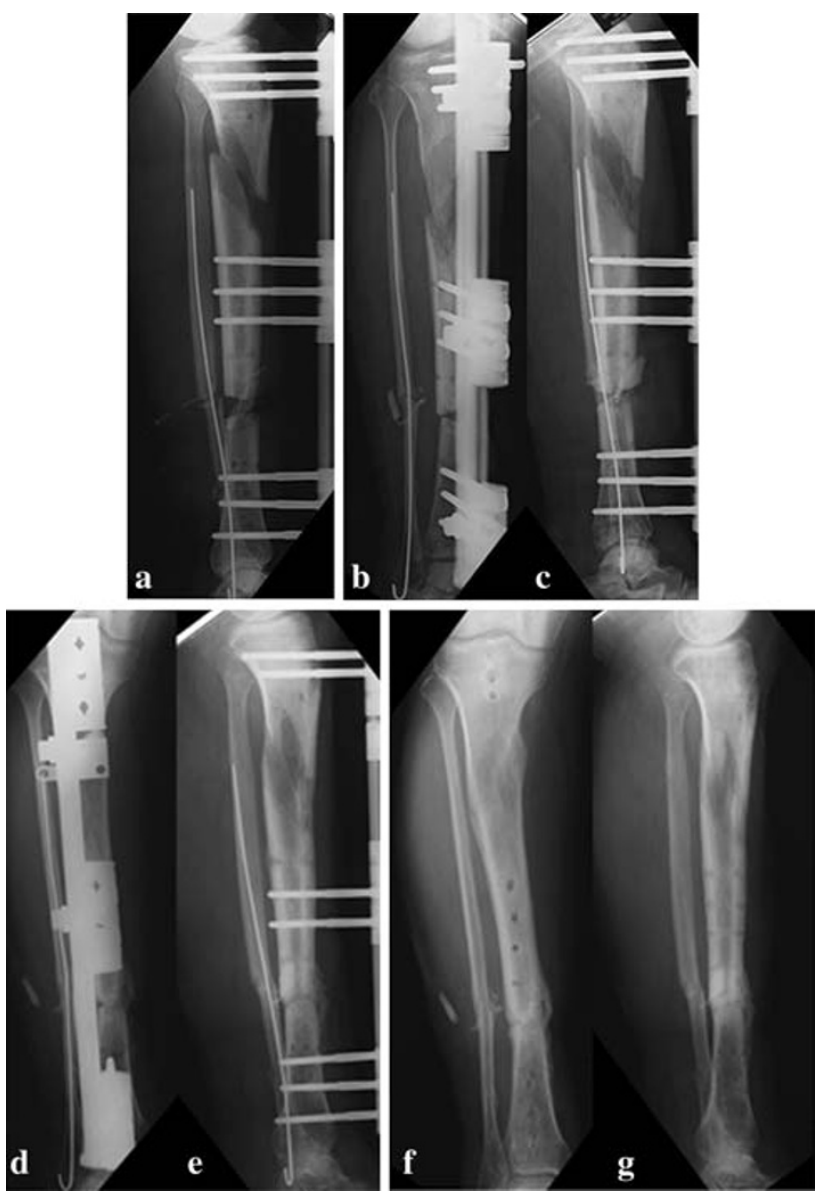

Fig. 2 V.O., man, aged 59. a Bone transport (descending technique) in previous proximal tibial closed fracture and distal exposed fracture with loss of bone. $\mathbf{b}$, $\mathbf{c}$ Check-up after second surgical examination of bone ends at point of contact. d, e Check-up 9 months, immediately after removal of fixator. $\mathbf{f}, \mathbf{g}$ Check-up 2 years after removal of fixator

commenced 5 days after osteotomy at an average rate of $1 / 3 \mathrm{~mm}$ every $8 \mathrm{~h}$.

Bone transport with the monolateral external fixator

The LRS rail fixator was applied using hydroxyapatitecoated screws: 3 proximal, 3 intermediate for transport and 3 distal. In cases where the resection margins could be determined pre-operatively and the focus of infection identified, the rail fixator was applied first prior to performing the resection. This facilitated maintenance of alignment between proximal, intermediate and distal bone segments. A "crown" osteotomy was performed in the healthy bone tissue to permit distraction in accordance with the callotasis technique [15]. Distraction in order to produce bone transport was started 10 days after surgery at a rate of $1 \mathrm{~mm} /$ day. 

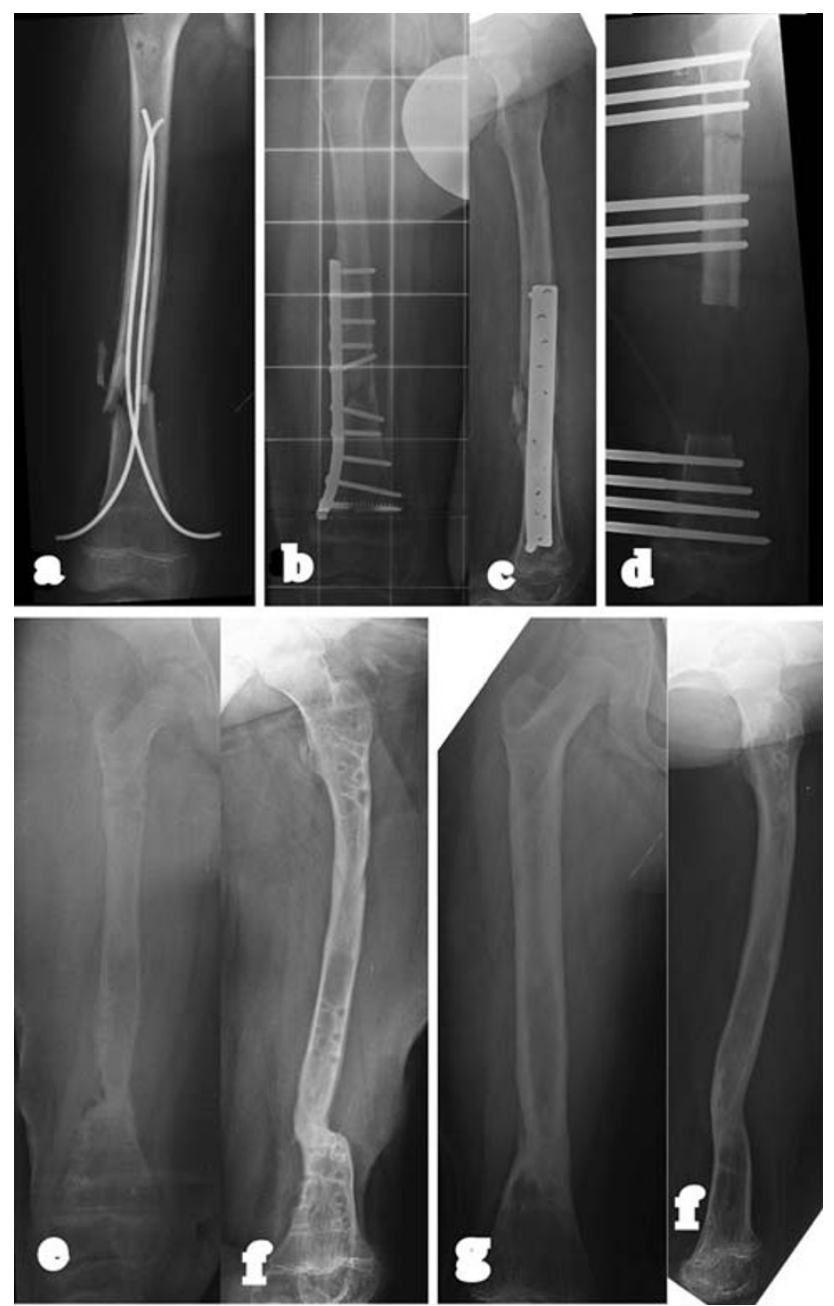

Fig. 3 N.A., man, aged 11. a-c Osteomyelitis secondary upon previous operations. d Check-up after resection, application of monolateral fixator and osteotomy for transport. e, f Check-up 8 months, immediately after removal of fixator. $\mathbf{g}$, h Check-up 2 years after removal of fixator

Common features to both fixator methods

The process of distraction osteogenesis is associated with an increase in the local blood supply to the limb. For example, in an open tibial fracture with bone loss which needed vascular reconstruction using a bypass graft, two arteriograms which were carried out before and after transport showed an improvement in the vascularization of the foot (Fig. 5).

The following factors in surgical technique were common to both groups irrespective of the type of fixator used:

1. The osteotomy of the bone ends was carried out in order to achieve good bone contact when the transported segment docked
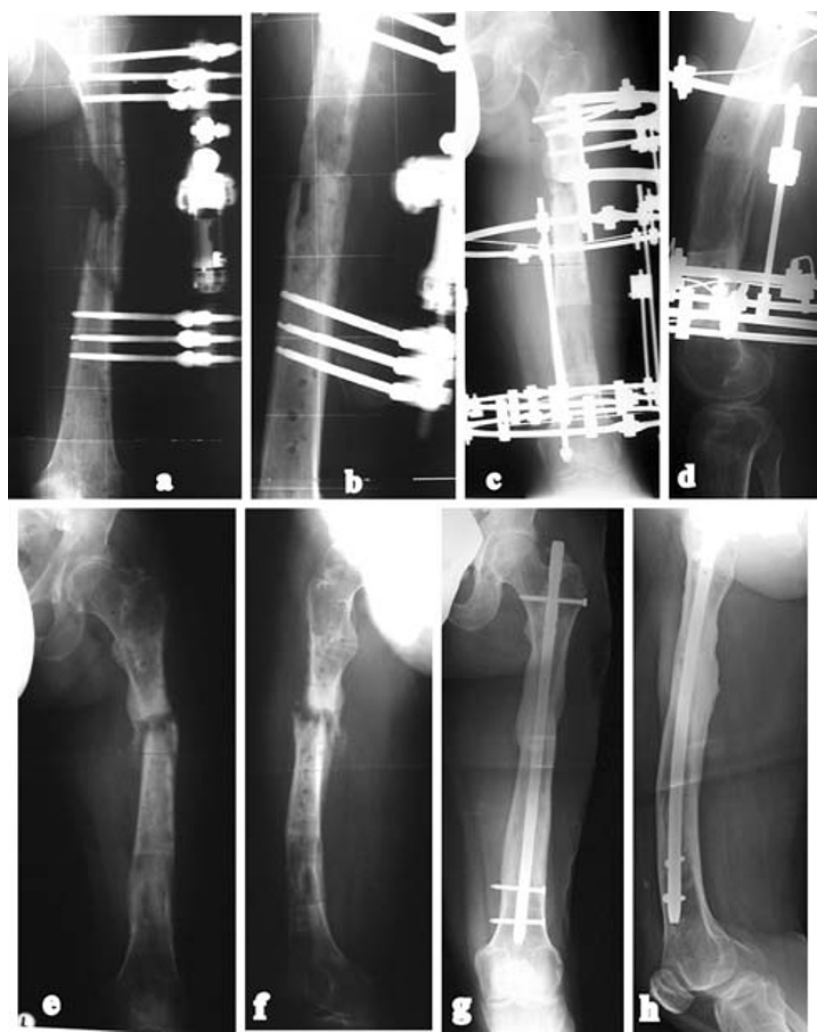

Fig. 4 C.A., man, aged 28. a, b Osteomyelitis after exposed fracture with bone gap, treated with external fixator and later osteosynthesis. c, d Check-up 7 months after transport. e, f Pseudoarthrosis at point of contact, 1 year after initial transport. g, h Check-up 3 years after nailing

2. Radical surgical debridement included removal of necrotic tissue until it was evident that the remaining bone ends bled

3. In all cases, samples were taken for culture. These included no fewer than 4 swabs-both before and during surgery. The latter sample was always more significant than the first as it was more likely to be representative of the causal bacteria in the deep infection. Subsequent antibiotic therapy was advised by specialists in infectious diseases. During surgery, a sample was also taken for histological examination.

4. We used ultrasound $[12,13]$ for cases where control $\mathrm{X}$-rays did not clearly show the presence of a regenerate column. This permitted earlier identification of the initial ossification before its presence could be detected by X-ray. At the end of transport, the contact point (docking site) was surgically cleaned, interposed tissue removed and, if small residual gaps were seen between the two bone ends, cancellous bone taken from the ipsilateral iliac crest and grafted. In 4 femurs, due to lack of consolidation and ossification of the regenerate column, we replaced the fixator with a intramedullary nail. 


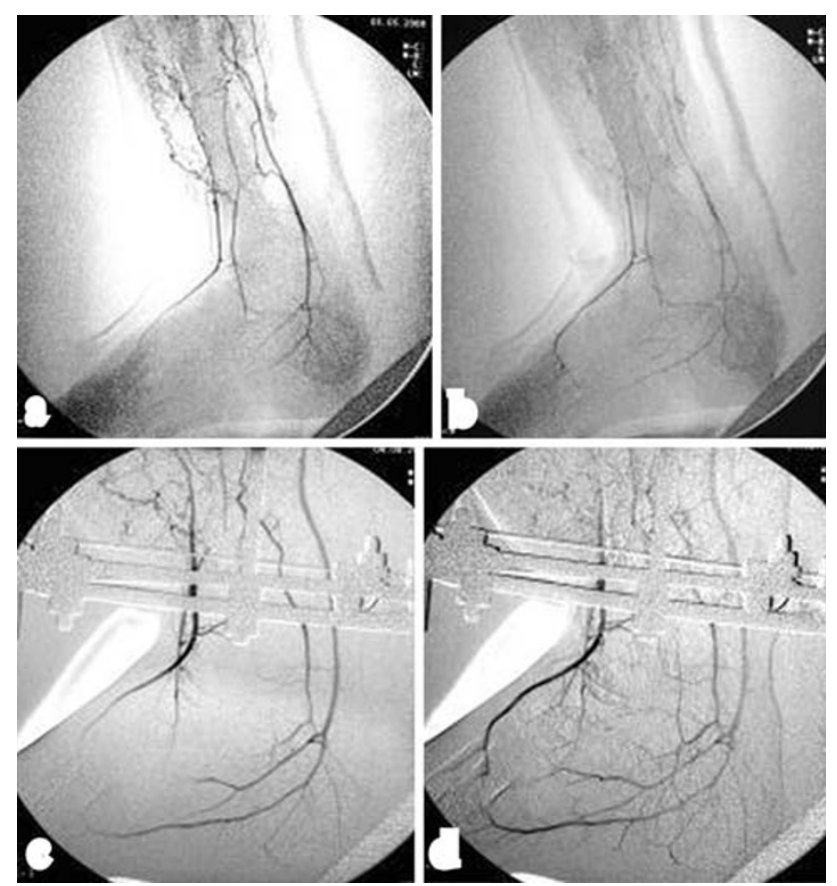

Fig. 5 F.L., man, aged 32. a, b Arteriography in an exposed tibial fracture with loss of bone. c, d Arteriography 3 months after transport shows improved vascularization of foot

\section{Results}

The process of bone transport is associated with problems, obstacles and complications [14]. We have chosen not to differentiate the difficulties encountered into these groups as we note all types to be clinically relevant. Including cases with complications, the total treatment time ranged from a minimum of 5 months to a maximum of 2 years. The average follow-up was 6 years (min. 2, max. 14). In 96 cases, bone transport led to normal neoformed bone. In our consecutive 100 cases, we observed a variety of complications known to be associated with the bone transport technique. These were as follows:

1. Failure of distraction osteogenesis (10/100 cases): The following additional procedures were necessary to restore limb integrity: 1 tibia healed with a second transport operation (osteotomy carried out on the opposite metaphysis); 3 tibias healed with compression followed by distraction of the transport segment (accordion); 1 femur healed with an autogenous bone graft; two femurs and 1 tibia were treated with intramedullary nailing; an amputation was performed for one depressed male patient who did not accept further reconstructive surgical solutions; and lastly, one man aged 62, who was non-compliant and who was treated with proximal-distal transport for a resection after an infected nonunion of the tibia, refused further treatment and preferred to use a brace for walking.

2. Refracture of the newly formed bone segment (1/100): This occurred at the proximal third of the femur and healed after the patient had used a cast for 50 days.

3. Premature consolidation in a segment of newly formed bone (1/100): This occurred in the proximal third of a tibia and was treated with a second osteotomy and repeated transport.

4. Deformity of the newly formed bone column (2/100): This occurred in 2 tibias and were only of a moderate degree ( 1 with $5^{\circ}$ recurvatum and 1 with 8 degrees procurvatum).

5. Nonunion at the docking site (17/100): In one femur and one tibia (both of which had already been treated with debridement and application of autoplastic grafts), this was managed with intramedullary nailing. The remaining 9 femurs and 6 tibias were successfully managed using a decortication technique applied to the docking site.

6. Recurring infection (4/100): All occurred in the tibia. Three cases were resolved with targeted antibiotic therapy; 1 case, with extensive osteomyelitis, required an amputation.

7. Knee stiffness (10/100): This occurred after femoral transport and all cases were resolved with a quadricepsplasty according to Judet.

\section{Discussion and conclusions}

This review allows us to confirm previously reported data and to highlight some aspects of the technique.

\section{Bone resection}

This is necessary to remove necrotic and infected bone and soft tissue. Resection must be radical and cross over into healthy tissue. In order to ascertain the vitality of the residual bone, it is best to drill several holes into the bone ends and observe bleeding with the tourniquet deflated. Samples of removed tissue should be examined both histologically and microbiologically. Collaboration with a specialist in infectious diseases is essential for appropriate antibiotic therapy.

\section{Osteotomy}

This was carried out with the "crown" technique in 45 cases and with a Gigli saw in 55 cases without significant differences in the quality of the newly formed bone. 
Bone transport

The transported segment of bone was almost always held with 3 screws in the axial fixator and with 2 in the circular. The extra screw is advisable in the monolateral fixator because it improves stability and reduces the risk of osteolysis. Although the diameter of the screws is greater than that of the tensioned wires, the screws were tolerated better. This is especially true of the tibia where the screws are inserted through the medial subcutaneous surface which not being covered by muscle is tolerant of the presence of screws.

In osteoporotic bone and where the bone ends need to be transported for less than $5 \mathrm{~cm}$, we found it preferable to increase the latency period after osteotomy to 10 days and reduce the rate to $0.5 \mathrm{~mm} /$ day, at least for the first 10 days. Ultrasound imaging will provide an indicator of the quality of osteogenesis and the possibility of accelerating distraction.

Contact point at end of transport (docking site)

In our opinion, this should always be explored surgically. In the light of the results obtained, and especially for the last 50 cases, we believe that, in the tibia, it is always necessary to remove interposed fibrous tissue and sometimes even cutaneous tissue (skin) whilst maintaining external fixation. Skin, especially in transport involving more than $3 \mathrm{~cm}$, caps the transported bone end. This needs to be freed and the bone ends carefully abraded with autoplastic grafts to accelerate consolidation. In these cases, especially those from a proximal-distal bone transport, the circular fixator gives better results as the disconnection of the two most distal rings allows reduction and optimal alignment of the two docking ends. This is then stabilised by re-connecting the rings. In the femur, we believe that the most effective method of solving nonunion of the docking site is by intramedullary nailing, this is with prior reaming of the medullary canal in order to remove all fibrous interposed growth and to prepare the site for reduction (Fig. 4).

\section{Shortening/lengthening}

An acute reduction of the size of bone defect by shortening the limb decreases treatment times because the two ends can be reduced and consolidate earlier. Shortening may reach $15 \%$ in the femur. The tibia cannot be shortened by more than $10 \%$ due to the risk of a compartment syndrome. In addition, resection of the fibula also requires further optimal corticalisation of the tibia before the fixator can be removed. However, this modification of the classic bone transport method is effective in the femur but should be avoided in the tibia. This is because it is preferable to maintain the continuity of the fibula except where trauma has already caused a gap of a few centimetres in the fibula.

Double transport

This can decrease bone transport times and is carried out by osteotomy of both proximal and distal femoral and tibial metaphyses and bringing the transported ends into contact with each other in the mid-diaphyseal region. Twin transport, with the bone ends both being taken in the same direction, is less effective. In the 4 cases of twin tibia transport, we encountered long corticalisation times of the newly formed regenerate bone from the more distal osteotomy, which is usually carried out in the diaphysis.

Practical factors with circular and monolateral fixators

Circular fixators are heavier and more uncomfortable for the patient, especially in assemblies on the thigh, and were found to be associated with longer medication times in this series. However, an advantage is that the assembly can be adjusted and modified without return to the operating room unless there is need for re-implantation of wires or screws during the transport procedure. Another advantage is in the tibia where, in proximal-distal bone transport, the two distal rings can be disconnected from the main assembly to allow access for surgical debridement of the docking site and for optimal reduction of the transported end to the distal tibia, and this followed by re-connection of the two rings. The experiences we have gathered in this series have led us to choose a monolateral fixator for femoral problems and the circular type for the tibia although we recognise that surgery should be planned with the possibility of using both types.

\section{Summary}

We present the results of 100 consecutive cases of bone transport with particular reference to the complications and their management. Bone transport is a reliable method for reconstruction of bone defects in the femur and tibia and remains a safe treatment for resection after bone infection. The prolonged treatment times and the need for repeated prescriptions for medication make this form of surgery inadvisable for the socially disadvantaged, those with poorly managed mental illness or those without a supportive family environment. Limb ablation by amputation is an alternative means of treatment and should be considered when local and patient-related factors indicate it is a preferred option. 


\section{References}

1. Aldegheri R, Berizzi A (2003) Trasporto osseo con fissatore monolaterale. Ortopedia e Traumatol s.e.r.t.o.t. 45:71-74

2. Aldegheri R, Iacobellis C, Berizzi A (2005) Trasporto osseo all'arto inferiore. Giorn Ital Ortop Traum 31(Suppl. 2):473-476

3. Aronson J (1992) Cavitary osteomyelitis treated by fragmentary cortical bone transportation. Clin Orthop Relat Res 280:153-159

4. Cattaneo R, Catagni M, Johnson EE (1992) EE The treatment of infected nonunions and segmental defects of the tibia by the method of Ilizarov. Clin Orthop Relat Res 280:143-152

5. De Bastiani G, Aldegheri R, Renzi-Brivio L (1979) Il fissatore esterno assiale. Chir Organi Mov 65:287-293

6. Dendrinos GK, Kontos S, Lyritsis E (1995) Use of the Ilizarov technique for treatment of non-union of the tibia associated with infection. J Bone Joint Surg Am 77:835-846

7. Green SA, Jackson JM, Wall DM, Marinow H, Ishkanian J (1992) Management of segmental defects by the Ilizarov intercalary bone transport method. Clin Orthop Relat Res 280:136-142

8. Iacobellis C (2003) Trasporti ossei con fissatore circolare. Ortopedia e Traumatol s.e.r.t.o.t. 45:75-80
9. Iacobellis C (1998) Le metodiche di Papineau e di Ilizarov a confronto nel trattamento delle osteiti posttraumatiche. Giorn Ital Ortop Traum 24:177-187

10. Mora R, Bertani B, Pedrotti L, Tuvo L, Gili S (2006) Compressiondistraction methods in nonunions of the long bones. In: Mora $\mathrm{R}$ (ed) Diagnosis and treatment with compression-distraction techniques. Springer, Milano. pp 89-117. ISBN: 978-88-470-0408-5

11. Paley D, Tetsworth K (1991) Percutaneous osteotomies. Osteotome and Gigli saw techniques. Orthop Clin North Am 22:613-624

12. Aldegheri R, Volino C, Zambito A, Tessari G, Trivella G (1993) Use of ultrasound to monitor limb lengthening by callotasis. J Pediatr Orthop 21:22-27

13. Iacobellis C, Bulzacchi A, Rioda A (2002) The ultrasound scan in monitoring regenerated bone. J Orthopaed Traumatol 3:41-48

14. Paley D (1990) Problems, obstacles and complications of limb lengthening by the Ilizarov technique. Clin Orthop Relat 250:81104

15. Aldegheri R, Renzi Brivio L, Agostini S (1989) The Callotasis method of limb lengthening. Clin Orthop Relat Res 241:135-137 